\title{
Meta-Heuristic based Adaptation Engine for Cognitive Radio Systems
}

\author{
Ramy A. Fathy \\ Digital Services Planning \\ Telecom Regulatory Authority \\ (NTRA), Egypt
}

\author{
Ahmed A. AbdelHafez \\ Electronics and Comm. Dept. \\ Military Technical College \\ (MTC), Egypt
}

\author{
Abdelhalim Zekry \\ Electronics and Comm. Dept. \\ Ain Shams University \\ (ASU), Egypt
}

\begin{abstract}
Cognition is a high level mental faculty of the brain that includes functions like adaptation, learning, deciding, and others. Accordingly, a Cognitive Radio must have capabilities that mimic such Cognitive functions. As one of the fundamental cognitive abilities of the radio, this paper proposes a novel adaptation method; which uses Real-coded Genetic Algorithms (RGA) to adapt physical layer radio parameters in response to varying environmental conditions and different user services. The adaptation method is applied in a single objective optimization setting - that's the minimization of BER. Minimum transmitted EIRP levels of the resulting solutions are achieved by using a special Power Limiting Algorithm (PLA) which increments the maximum transmitted allowable EIRP levels during the engine run, if it experienced a slow convergence towards the optimal required solution. Results have indicated the success of the engine in adapting the physical layer radio parameters in response to varying environmental conditions and different user services to minimize resulting link BER, with the minimum possible transmitted EIRP levels.
\end{abstract}

\section{General Terms}

Cognitive Radio, Meta-heuristics, Wireless Communication.

\section{Keywords}

Adaptation Cycle, Adaptation Engine, Real-coded Genetic Algorithms.

\section{INTRODUCTION}

The introduction of Cognitive Radio (CR) by Mitola in 1999 [1], represents a core technology that enables service providers, operators and National Regulatory Authorities to migrate away from the Command and Control regime; towards more open access spectrum policies [2]. This is an important step towards accessing the spectrum dynamically; and hence maximizing its usage. CR has shifted the paradigm of communications systems designers; in focusing on other technical solutions from other scientific paradigms, to tackle traditional problems.

Basically a CR have three basic functionalities for cognitive behavior: sensing the spectrum and the environment surrounding the radio, the ability to represent and comprehend knowledge and act accordingly through a capacity to reason and learn; and finally the ability to adapt its operating parameters in respond to varying stimuli [1]. In this paper, we focus on the ability of the radio terminal to adapt its physical layer parameters to its environment. Adaptation involves introducing adaptive algorithms that enable the radio terminal from achieving higher spectrum utilization, and better link performance.
In [3] the authors use BER curves to initialize their Genetic Algorithm (GA), in an attempt to reduce their BER; in a single objective utility function setting. In their design, communications principles like Bit Error Rate (BER) and capacity curves were used to improve the engine's learning rate.

Another attempt to use GA was done by [4] to optimize a wireless link, using however a multi-objective utility function based also on communications principles. The engine defined a normalized multi-objective utility function summed into one, with three main objectives: minimizing BER, minimizing transmission power, and maximizing the throughput.

On the other hand, the authors in [5] designed and implemented a cognitive radio adaptation engine using Particle Swarm Optimization (PSO). They applied the engine in a multi-objective optimization setting where minimum transmitted power, minimum achievable BER, and maximum throughput goals are traded against each other based on the application.

The primary goal of this paper is to present a novel design and implementation of an Adaptation Engine (AE) that is based on meta-heuristic techniques. Real-coded Genetic Algorithm (RGA) is used as the core technique in the engine implementation due to its inherent parallelism, in addition to its ability to solve highly complex multi-dimensional optimization problems without the need of supervision, i.e. autonomous.

The rest of this paper is organized as follows: Section 2 illustrates the CR adaptation cycle. Section 3 presents and details the proposed $\mathrm{AE}$ architecture based on Real-coded Genetic Algorithm (RGA). It also presents the proposed implementation of the engine and some practical considerations related to the implementation. Section 4 presents our experimentation and validation procedures for the accuracy and reliability of our implemented engine. Section 5 demonstrates and discusses the results. Finally Section 6 presents the conclusion and future work that will be addressed in our research.

\section{THE COGNITIVE RADIO ADAPTATION CYCLE}

The characterization of the $\mathrm{CR}$ adaptation cycle provides the main states of operation of the engine. The adaption cycle clearly demonstrates the conditions of operation of each state in addition to the actions the engine undertakes under varying environmental conditions and link states.

Our proposed CR system model, assumes a typical scenario where two SDR-based transceivers equipped with a Cognitive Radio Engine (CRE) and Sensing Modules communicate in a 
hostile environment where unknown interference and background noise sources are present in the vicinity of the transceivers. The channel characteristics and channel response are not known a priori to the commencement of the communication process.

Subset of the CRE is the Adaptation Engine (AE) which performs adaptation functions by employing meta-heuristic algorithms and techniques to dynamically adapt the radio's internal operations to meet certain objectives inferred by the CRE.

The CR adaptation function operates in an adaptation cycle as shown in Figure 1. At the start of the operation of any CR, there is no established prior link established between any two transceivers. A CR enters into Link Setup Phase where the radio discovers its ambiance by scanning for any band activity and/or another terminal. In this state a protocol is followed by the radio terminal to establish a communication link with another one; through the exchange of primary configuration information and other control messages (rendezvous problem). A possible straightforward method to conduct rendezvous is to exchange these primary configuration information and other control messages on a preconfigured initial control channel.

The CR then waits for user input regarding which terminal to contact and what service to engage into. According to the service selection, the $\mathrm{CR}$ enters into an Initialization Phase where the $\mathrm{AE}$ performs an initial adaptation for the link parameters to satisfy the best link configuration that would minimize the BER, for the service selected by the user at the end of the Setup Phase. The terminal initiating transmission is assumed to take control over the recipient in a Master-Slave configuration to avoid uncontrolled ping-pong between the two transceivers.

Spectrum Sensing enables the terminal of acquiring band activity in addition to the interference plus background noise levels from the surrounding environment. After determining the best solution representing the optimum set of operating parameters given the channel conditions, interference and noise levels, and preferred service from the user; the system undergoes multiple feedback loops by sending a specially formatted integrity checking test messages for limited period of time to account for any possible differences between the calculated performance metrics (BER in this case) and the measured ones after real transmission. That coincides with other observations stating that CRs are, by necessity, an example of feedback communications systems [6].

Having configured the link for the optimum configuration, the communication process could be initiated after going into the Idle State. An alternate possibility to overcome the potential session establishment time that could arise during the Initialization Phase without the start of the communication process, is to start communicating immediately as soon as a link is established (after the rendezvous) and a service is selected using the initial link configuration before the initial adaptation performed during the Initialization Phase. This has the effect of reducing the session establishment time at the expense of the probable quality degradation of the established link. This is because rendezvous usually establishes a link without optimizing the link for specific performance targets.

Specific adaptation cycle implementations depend on the target application of the CR. For example, an assertion on highest quality of the link regardless of the session establishment time might be more important in disaster recovery and extreme conditions situations; and this is our main targeted goal in our research

After initializing the two transceivers and reaching to a common configuration that bests suits the channel for a certain objective, the $\mathrm{AE}$ enters into the Idle State, where normal communication procedures can proceed. The master transceiver sends and receives messages to and from the slave transceiver as in any normal communication setup. The adaptation cycle demonstrates the action the radio will undertake under two environmental situations as shown in the following sub-sections.

\subsection{Link Drop}

Link drop is defined as the decrease of the SNR at the receiver below a certain threshold and for a considerable amount of time. Another possible indication for the link drop is that the decrease in the SNR is sustainable or persistent; that's the decrease in the performance is increasing.

This is expressed mathematically by the transition condition of the Adaptation Cycle - See Figure 1 - presented in (1) as follows:

SNR $<$ SNR Threshold,

and

$\frac{d(S N R)}{d t}<0$,

and

$t_{n}-t_{\text {drop }}>t_{\text {thresh }}$

where SNR Threshold indicates the threshold below which the link quality is considered unacceptable, and $t_{n}, t_{d r o p}, t_{t h r e s h}$ refers to time at instant $n$; and time of link drop; and time threshold after which the link is considered to be persistently dropping respectively.

If the link degrades, communication might become impossible between the two transceivers. However, it is highly unlikely that the transceivers would stop their inter-communication and return to the Initialization Phase. Since there is a probability that the Initialization Phase might take some time depending on the nature of the impairment; which might cause a lot of inconvenience for the users. Therefore, if the link degrades, the transceivers enter into the Adaptation Phase where performance metrics - Received Signal Strength (RSS), in our case - are fed back from the slave to the master transceiver along with the communicated messages to enable the $\mathrm{AE}$ of the master to optimize again the operating parameters in a way that achieves a certain utility function depending on some channel performance metrics.

Sending performance metrics along with the original messages decreases the overall throughput. However this is a cost we might be willing to pay in order to maintain the communication link without breaking up the communication process. Again after reaching a common configuration suitable for the new channel conditions, the Communication Phase can resume as normal.

\subsection{Operating Frequency Band Unavailability}

If the operating frequency band on which the link was established becomes unavailable, possibly due to the reappearance of the primary user, or an interferer using that channel; an immediate vacation for the band is necessary. However, searching for solutions (best parameters set) which includes a suitable operating frequency; that would eventually 
achieve the targeted objective(s) could be a time consuming processing especially if meta-heuristic techniques are used. Hence, it would be extremely difficult to commence the solution search process, when such a situation arises.

Therefore, in our design; the following strategy is used:

a) when the $\mathrm{AE}$ searches for optimum solutions to achieve some objective(s), it is possibly to find the best, second best, third best and so on.. for the problem under study;

b) when the $\mathrm{AE}$ finds an optimum/sub-optimum solution; it sets the internal parameters of the radio on this new parameters set;

c) at the same time, and through a continuous process of spectrum sensing, the radio senses the different bands for a probable appearance of primary users in the newly set band of operation;

d) as soon as a primary user is detected; the frequency sensor senses the vacancy of the band belonging to the second best solution identified by the engine; if this band is free, the evacuation could start immediately; without the need to run the $\mathrm{AE}$ once more to find an optimum solution;

e) finally the performance is monitored and the CR could enter into the Adaptation Phase if a degradation of performance is detected just as in normal operation of the system.

\section{ADAPTATION ENGINE \\ ARCHITECTURE BASED ON REAL- CODED GENETIC ALGORITHM (RGA)}

In the previous section, the adaptation cycle of the $\mathrm{AE}$ is presented. In this section, the proposed $\mathrm{AE}$ architecture employing meta-heuristic techniques using real-coded GA is presented. Instead of modeling the channel or trying to analyze its effects and artifacts on the received signal, a CR doesn't have to model or analyze the behavior of the channel. It relies however on the $\mathrm{AE}$, a subset of the CRE which employs meta-heuristic algorithms and techniques to dynamically adapt the radio's internal operations to meet certain objectives inferred by the CRE from the goals of the radio and its surrounding operating context.

Genetic algorithms (GA) are optimization methods that mimic natural evolution. Optimization is based on the development of the population comprising a certain number of chromosomes. The chromosomes represent a possible solution set for the optimization problem; which could be maximization or a minimization for a specific objective function. The population size indicates the number of parallel solutions that would be tried in parallel to reach towards the optimum/sub-optimum solution. The development of the population is regulated by means of two genetic operators; namely, crossover and mutation [7].

\subsection{Real-coded Genetic Algorithm (RGA)}

The core of genetic algorithms involves the encoding of probable solutions to the optimization problem as arrays of bits or character strings to represent the chromosomes; the manipulation operations of these encoded solutions (chromosomes) to produce offspring, by means of genetic operators; and the selection according to their fitness with the aim to find a solution to the problem concerned.
A traditional binary-coded GA [8] is based on encoding the solutions into binary strings, where each chromosome is represented by a string of binary digits. The crossover and mutation genetic operators are relatively simple. However, essential to the operation of the algorithm is the binary to decimal conversion procedure for substituting the values corresponding to the binary-coded chromosomes in the utility function to determine the fitness of the solutions. The binary to decimal conversion procedures are done for every chromosome in the population; which might cost time and computations.

Alternatively, in our approach, real-coded GA (RGA) is used as the core of the AE. In order to reduce the amount of computations, and the dynamic response of the engine; the binary to decimal or decimal to binary conversions have been eliminated, by employing RGA. Chromosomes are not expressed as binary strings anymore. They are directly initialized as real values, and special techniques have been applied for conducting crossover and mutation operators using real-coded chromosomes.

However, this approach also suffers from slightly more complexity in the implementation of the genetic operators as compared to the binary-coded situation. The following subsections will highlight the crossover and mutation genetic operators that are implemented in the RGA based AE.

\subsection{Crossover Method}

Crossover genetic operator is one of the fundamental genetic operators in GA. Crossover consists of swapping chromosome parts, at one or more random points, between two pairs of individuals in the population to produce new offspring. The crossover frequency is controlled by the parameter $P_{c}$; the probability of crossover.

Mathematically, parents are denoted by (2):

$x^{(1)}=\left(x_{1}^{(1)}, \ldots, x_{n}^{(1)}\right)$

$x^{(2)}=\left(x_{1}^{(2)}, \ldots, x_{n}^{(2)}\right)$

While the resulting offspring after the crossover genetic operation is expressed in (3) as:

$y^{(1)}=\left(y_{1}^{(1)}, \ldots, y_{n}^{(1)}\right)$

$y^{(2)}=\left(y_{1}^{(2)}, \ldots, y_{n}^{(2)}\right)$

where $x^{(i)}$ represents the $i^{\text {th }}$ parent, while $n$ represents the number of genes in the chromosome.

Crossover in real-coded GA can be performed by many methods like Arithmetic Crossover (AMXO), Heuristic Crossover, and Parent-Centric Crossover [9, 10].

The AMXO method has been implemented due to its simplicity. The AMXO method can be illustrated mathematically by (4) as follows:

$y_{i}^{(1)}=\alpha_{i} x_{i}^{(1)}+\left(1-\alpha_{i}\right) x_{i}^{(1)}$,
$y_{i}^{(2)}=\alpha_{i} x_{i}^{(2)}+\left(1-\alpha_{i}\right) x_{i}^{(2)}$,

where $\alpha_{i}$ are uniform random numbers $[9,10]$. 


\subsection{Mutation Method}

Mutation aids GA from escaping local minima by introducing new genetic material in the population so as to maintain the population diversity. Mutation is achieved by randomly changing some gene(s) in the chromosome of an individual. Mutation is regulated with the mutation probability, $P_{m}$. Before mutation, the chromosome can be mathematically denoted by (5):

$x=\left(x_{1}, \ldots, x_{i}, \ldots, x_{n}\right)$

where the gene selected for mutation is denoted by $x_{i}$.

After mutation, the chromosome can be mathematically expressed as in (6):

$x^{\prime}=\left(x_{1}, \ldots, x_{i}^{\prime}, \ldots, x_{n}\right)$

Mutation in RGA can be performed by many methods like Mäkinen, Periaux and Toivanen mutation (MPT), Power Mutation, and Boundary Mutation [11]. We have implemented the MPT mutation method which can be illustrated mathematically by (7), (8), and (9) as follows:

$x_{i}^{\prime}=(1-\tau) l b_{i}+\tau u b_{i}$,

where,

$\tau= \begin{cases}t-t\left(\frac{t-\operatorname{rand}()}{t}\right)^{\text {beta }}, & \text { if } \operatorname{rand}()<t \\ t, & \text { if } \operatorname{rand}()<t \\ t+(1-t)\left(\frac{\operatorname{rand}()-t}{1-t}\right)^{\text {beta }}, & \text { if } \operatorname{rand}()>t\end{cases}$

and,

$t=\frac{x-l b_{i}}{u b_{i}-l b_{i}}$

such that,

$l b_{i}$ and $u b_{i}$ are the lower and upper bounds of the $i^{t h}$ decision variable of each gene respectively; and $\operatorname{rand}()$ is a uniformly distributed random number $\in[0,1]$

\subsection{Implementation}

The AE is implemented and tested using MATLAB version 7.0.0.19920 (R14). Figure 2 illustrates our proposed AE architecture. The engine is designed to generate a number of equiprobable random frequency values (equal to the preset number of individuals inside the population) between the minimum and maximum sensor frequency operating ranges in accordance to the hardware capabilities of the frequency sensor. Same applies for the transmitter power and modulation types used.

The AE starts by generating a number of random possible solutions belonging to the solution space; which includes different combinations of frequency of operation, the transmitter's transmitted power, and the modulation/demodulation that are going to be used in the communication process. The engine is constrained not to operate beyond a maximum adjustable operating transmitted EIRP, so as to minimize the power consumption of the radio terminal to the maximum extent possible. A special Power Limiting Algorithm (PLA) is developed to use the lowest possible transmitted EIRP while only a single goal is targeted; that's minimizing the BER. A possible power level constraint could be fed from a Policy Engine, a module which belongs to the CRE; responsible of identifying among others, the maximum allowable power levels in every band in accordance to regulatory constraints.

The possible solutions are encoded (real encoding) into a number of chromosomes which represent the set of possible candidate solutions for the optimization problem. A percentage of best fit chromosomes are selected for reproduction based on their fitness. Those selected chromosomes replace less fit individuals from previous generations. The process continues till a certain level of accuracy is achieved or a maximum number of generations are exceeded.

The Frequency Sensor module continuously senses the spectrum for vacant frequency bands and noise plus interference levels in these bands. This information is passed to the AE through the Dynamic Frequency Occupancy Map and Noise plus Interference Samples. The former, is a map which identifies a temporal snapshot of unoccupied frequency bands which are suitable for transmitting the services selected by the user.

In the reproduction phase, the resulting offspring (candidate solutions) are checked to be valid; that's to belong to the feasible solution space. Evolution follows when the fitness of the offspring is greater than those the individuals in the prior generations but were not part of the reproduction process. This process is continued till a solution with a certain level of accuracy is found or a maximum number of generations are surpassed.

\section{EXPERIMENTATION AND VALIDATION PROCEDURES}

By implementing the AE using MATLAB, an experimentation procedure has been conducted to validate the accuracy of the engine's resulting solutions. In general the validation procedures target two main objectives:

a) Accuracy Validation: to ensure the solutions' accuracy of the AE to the optimization problem;

b) Performance Assessment: by measuring the Engine Dynamic Response Time

The test is conducted for many runs to ensure statistical confidence, especially since GA has a stochastic nature, especially in its core elements, e.g. selection, and genetic operators

\subsection{Experimentation}

To validate the accuracy of the results of the AE; we have conducted an experimentation procedure using six Test Cases as a basis for evaluation. Figure 3 demonstrates the different Test Cases used for the evaluation of our proposed AE. The idea behind the Test Cases is to deploy the AE in multiple scenarios of bands occupancy, and different noise plus interference levels for low (1 Mbps) and high (10 Mbps) service scenarios; to observe the resulting engine's solutions, and test it in a test bench. The test bench is developed where mathematically pre-calculated solutions for the Test Cases is established for comparing its results with the engine results.

All the noise plus interference profiles used in the experimentation procedure are acquired through exporting I/Q data of the amplitude signal strength of SA2600 Handheld Spectrum Analyzer; into CSV and MATLAB formats for further processing. The CSV and MATLAB I/Q format signal 
strengths files are entered to the engine as noise plus interference samples at specific resolution depending on the bandwidth required for the service as explained above in the Test Cases. The SA2600 Handheld Spectrum Analyzer connected to Omni-directional antenna; is used to simulate for the noise plus interference levels supplied by the Frequency Sensor in a typical CRS. This method is used to test the engine response in real scenarios that are found in the field.

To simulate the presence of interference above the normal noise floor (ranging from $-115 \mathrm{dBm}$ to $-120 \mathrm{dBm}$ according to the resolution bandwidth), due to man-made noise sources, and others sources of interference; a signal generator generating a tone at specific frequencies inside the simulated bands are transmitted at low power levels with a maximum of $(\sim-104.22 \mathrm{dBm})$, using a directional antenna with antenna gain of $(7 \mathrm{dBi})$. The directional antenna used is then directed in close proximity (several meters) towards the omnidirectional antenna connected to the Spectrum Analyzer which acts in this setup as the Frequency Sensor of our CR. The interfering tone will result in the rise of the noise floor according to the amount of interference injected. A maximum noise plus interference levels are simulated in bands $820 \mathrm{MHz}$ $-831.25 \mathrm{MHz}$ which reached to $(-102 \mathrm{dBm})$ in Test Cases 5, and 6 . The resulting noise profiles are shown schematically in Figure 3.

\subsection{Accuracy Validation}

By using the test bench to mathematically pre-calculating the different solution sets accounting for different transmitted EIRP levels, modulation types, and inter-CR terminals distances; for the different Test Cases, a baseline for validating the accuracy of the engine is obtained.

Accuracy of the engine results is validated by cross checking the resulting solutions with the possible solutions spaces produced by the pre-calculated mathematical baseline. If an engine's solution lies within the set of acceptable solutions generated by the test bench baseline; then that solution is deemed accurate and acceptable.

\section{RESULTS}

This section represents the results of the various experiments conducted to validate the accuracy of our proposed AE. By running the $\mathrm{AE}$, the engine produces solutions that fall within the domain of possible solution spaces produced by the test bench. Figure 4 demonstrates a sample of the engine solutions for Test Case 6. A total of 14 solution sets resulted in BPSK waveform with varying EIRP levels and operating frequencies while another 67 solution sets resulted in QPSK waveform with varying EIRP levels and operating frequencies. Using Power Limiting Algorithm (PLA) enables the engine from finding the optimum solution autonomously during the engine runtime. However that is traded off with a higher Engine Dynamic Response Time as demonstrated below.

In addition the reliability of the engine's resulting solutions is determined by the percentage of time the engine produces correct solutions at different maximum transmitted EIRP levels. Table 1 presents the reliability assessment results for the different Test Cases presented in Figure 3, at different selected maximum allowable EIRP levels; and at an inter-CR terminals distance of $500 \mathrm{~m}$. Table 2 on the other hand demonstrates the resulting engine's reliability and performance.

Results demonstrate that as the span of the available frequency options increases; the probability of generating correct solutions (i.e. the reliability) decreases for the same size of the population. In addition, the Power Limiting Algorithm (PLA) does enable the engine from finding the optimum parameter combinations (with varying levels of reliability) that minimized the link BER with the minimum of the greatest possible transmitted EIRP levels. However this comes at the expense of a higher mean Engine Dynamic Response Time as shown in Table 2. To enhance the reliability of the generated results when PLA is applied, two possibilities could be applied:

a) Increasing the Converging Threshold at the expense of higher Engine Dynamic Response Time.

b) Increasing the Power Step at the expense of a higher maximum allowable transmitted EIRP level if the step was higher than the minimum optimum EIRP level for the scenario surrounding the radio.

Adjusting the engine control parameters like increasing the population size, and/or the probability of mutation and crossover; does also have its impact on enhancing the reliability and accuracy of the solutions. The main engine control parameters used in this work are shown in (10) as follows:

$\begin{array}{ll}\text { Population Size } & =40 \\ \text { Probability of Crossover } P_{c} & =0.85 \\ \text { Probability of Mutation } P_{m} & =0.05 \\ \text { Maximum Number of Generations } & =100\end{array}$

Table 1 illustrates that increasing the frequency span of the $\mathrm{CR}$ in high data rate service deployment situations, has a much higher negative effect on the engine reliability compared to low data rate service deployment scenarios. On the other hand, increasing the maximum allowable EIRP levels of the solutions by possibly small ranges could lead to tremendous performance enhancement.

Table 1. Reliability Assessment at Different Selected Maximum Allowable EIRP Levels

\begin{tabular}{|c|c|c|c|c|c|c|}
\hline & & & \multicolumn{4}{|c|}{$\begin{array}{c}\text { Probability of Correct } \\
\text { Solutions (\%) }\end{array}$} \\
\cline { 4 - 7 } Test Case & $\begin{array}{c}\text { Max } \\
\text { \# Sub- }\end{array}$ & $\begin{array}{c}\text { \# } \\
\text { Engine } \\
\text { Bands }\end{array}$ & Runs & \multicolumn{4}{|c|}{$\begin{array}{c}\text { Selected Maximum } \\
\text { Allowable EIRP }\end{array}$} \\
\cline { 4 - 7 } & & & $\begin{array}{c}\mathbf{1 0} \\
\mathbf{d B}\end{array}$ & $\begin{array}{c}\mathbf{1 3} \\
\mathbf{d B}\end{array}$ & $\begin{array}{c}\mathbf{2 3} \\
\mathbf{d B}\end{array}$ & $\begin{array}{c}\mathbf{3 0} \\
\mathbf{d B}\end{array}$ \\
\hline $\begin{array}{c}\text { Test Case } \\
1\end{array}$ & & & & & & \\
$\begin{array}{c}375 \mathrm{KHz} \\
\text { Sub-bands }\end{array}$ & 23 & 100 & 92 & 99 & 100 & 100 \\
\hline $\begin{array}{c}\text { Test Case } \\
2\end{array}$ & & & & & & \\
$\begin{array}{c}3.75 \mathrm{MHz} \\
\text { Sub-bands }\end{array}$ & 3 & 100 & 0 & 0 & 79 & 100 \\
\hline $\begin{array}{c}\text { Test Case } \\
3\end{array}$ & & & & & & \\
$\begin{array}{c}375 \mathrm{KHz} \\
\text { Sub-bands }\end{array}$ & 44 & 100 & 88 & 98 & 100 & 100 \\
\hline $\begin{array}{c}\text { Test Case } \\
4\end{array}$ & & & & & & \\
$\begin{array}{c}3.75 \mathrm{MHz} \\
\text { Sub-bands }\end{array}$ & 5 & 100 & 0 & 0 & 73 & 100 \\
\hline
\end{tabular}




\begin{tabular}{|c|c|c|c|c|c|c|}
\hline & & & \multicolumn{3}{|c|}{$\begin{array}{c}\text { Probability of Correct } \\
\text { Solutions (\%) }\end{array}$} \\
\cline { 4 - 7 } Test Case & $\begin{array}{c}\text { Max } \\
\text { \# Sub- }\end{array}$ & $\begin{array}{c}\text { \# } \\
\text { Eangine } \\
\end{array}$ & & \multicolumn{4}{|c|}{$\begin{array}{c}\text { Selected Maximum } \\
\text { Allowable EIRP }\end{array}$} \\
\cline { 4 - 7 } & & & $\begin{array}{c}\mathbf{1 0} \\
\mathbf{d B}\end{array}$ & $\begin{array}{c}\mathbf{1 3} \\
\mathbf{d B}\end{array}$ & $\begin{array}{c}\mathbf{2 3} \\
\mathbf{d B}\end{array}$ & $\begin{array}{c}\mathbf{3 0} \\
\mathbf{d B}\end{array}$ \\
\hline $\begin{array}{c}\text { Test Case } \\
5\end{array}$ & & & & & & \\
$\begin{array}{c}375 \mathrm{KHz} \\
\text { Sub-bands }\end{array}$ & 59 & 100 & 31 & 73 & 100 & 100 \\
\hline $\begin{array}{c}\text { Test Case } \\
6\end{array}$ & & & & & & \\
$\begin{array}{c}3.75 \mathrm{MHz} \\
\text { Sub-bands }\end{array}$ & 7 & 100 & 0 & 0 & 0 & 85 \\
& & & & & & \\
\hline
\end{tabular}

For example, increasing the frequency span by $63 \%$ in low data rate service deployment scenarios; results in a reliability percentage decrease of $35 \%$ if the maximum allowable EIRP level were set at $10 \mathrm{~dB}$. However, increasing the maximum allowable EIRP levels increased from $10 \mathrm{~dB}$ to $13 \mathrm{~dB}$ results in a reliability percentage decrease of only $13 \%$. However, low data rate services does show worse mean Engine Dynamic Response Time compared to high data rate service deployment scenarios for the same maximum allowable EIRP level.

Table 2. Reliability and Performance Assessment of the AE

\begin{tabular}{|c|c|c|c|c|}
\hline $\begin{array}{c}\text { Test Case } \\
\text { Number }\end{array}$ & KPI & $\begin{array}{c}\text { Dynamic } \\
\text { Response } \\
\text { Time } \\
\text { (Sec) }\end{array}$ & $\begin{array}{l}\text { Maximum } \\
\text { Allowable } \\
\text { EIRP (dB) }\end{array}$ & $\begin{array}{c}\text { Reliability } \\
(\%)\end{array}$ \\
\hline \multirow{5}{*}{$\# 1$} & Mean & 0.88 & 10.12 & \multirow{5}{*}{97} \\
\hline & Median & 0.84 & 10.00 & \\
\hline & Std. Dev. & 0.16 & 0.59 & \\
\hline & Min. & 0.81 & 10.00 & \\
\hline & Max. & 1.67 & 13.00 & \\
\hline \multirow{5}{*}{$\# 2$} & Mean & 2.44 & 21.88 & \multirow{5}{*}{84} \\
\hline & Median & 2.45 & 22.00 & \\
\hline & Std. Dev. & 0.34 & 2.04 & \\
\hline & Min. & 1.90 & 19.00 & \\
\hline & Max. & 3.46 & 28.00 & \\
\hline \multirow{5}{*}{$\# 3$} & Mean & 1.11 & 10.21 & \multirow{5}{*}{91} \\
\hline & Median & 1.03 & 10.00 & \\
\hline & Std. Dev. & 0.29 & 0.88 & \\
\hline & Min. & 1.00 & 10.00 & \\
\hline & Max. & 3.09 & 16.00 & \\
\hline \multirow{3}{*}{$\# 4$} & Mean & 2.66 & 22.57 & \multirow{3}{*}{74} \\
\hline & Median & 2.60 & 22.00 & \\
\hline & Std. Dev. & 0.36 & 2.16 & \\
\hline
\end{tabular}

\begin{tabular}{|c|c|c|c|c|}
\hline $\begin{array}{l}\text { Test Case } \\
\text { Number }\end{array}$ & KPI & $\begin{array}{c}\text { Dynamic } \\
\text { Response } \\
\text { Time } \\
\text { (Sec) }\end{array}$ & $\begin{array}{l}\text { Maximum } \\
\text { Allowable } \\
\text { EIRP (dB) }\end{array}$ & $\begin{array}{c}\text { Reliability } \\
(\%)\end{array}$ \\
\hline & Min. & 2.01 & 19.00 & \\
\hline & Max. & 3.15 & 25.00 & \\
\hline \multirow{5}{*}{$\# 5$} & Mean & 2.61 & 12.19 & \multirow{5}{*}{80} \\
\hline & Median & 2.96 & 13.00 & \\
\hline & Std. Dev. & 1.04 & 2.13 & \\
\hline & Min. & 1.50 & 10.00 & \\
\hline & Max. & 4.63 & 16.00 & \\
\hline \multirow{5}{*}{$\# 6$} & Mean & 3.75 & 27.64 & \multirow{5}{*}{81} \\
\hline & Median & 3.79 & 28.00 & \\
\hline & Std. Dev. & 0.37 & 1.97 & \\
\hline & Min. & 3.18 & 25.00 & \\
\hline & Max. & 4.99 & 34.00 & \\
\hline
\end{tabular}

6. CONCLUSION AND FUTURE WORK

In this paper, a novel design and implementation for a RGA based $\mathrm{AE}$ that adapts its physical layer parameters to its environment have been presented. It is the first time to our knowledge that RGA is used in the adaptation function of cognitive radio $\mathrm{AE}$. To maximize the applicability and usefulness of our proposed $\mathrm{AE}$, the system is applied on a single objective utility function setting, with the goal of minimizing BER while achieving the minimum greatest transmitted EIRP levels to reduced power consumption. This is achieved through using a Power Limiting Algorithm (PLA) which increments the maximum transmitted allowable EIRP levels during the engine run, if it experienced a slow convergence towards the optimal required solution is developed to use the lowest possible transmitted EIRP while maintaining only a single goal (minimizing BER).

Results indicate a negative correlation between the number of available frequency bands and the reliability of the engine. A negative correlation is also observed between the data rate of the service to be provided and the reliability of the engine. Reliability refers to the ability of the engine to find correct solutions whenever it operates. Lower maximum allowable transmitted EIRP levels or higher data rate services have the tendency to limit the number of available solutions present in a specific band given certain in-band interference plus noise levels. This in turn affects the stochastic ability of the engine to produce correct solutions under heavy constraints, unless these constraints (very large frequency span, low maximum allowable transmitted EIRP, or large data rate services) are eased out.

Other factors that affect the accuracy and reliability of the engine are the engine control parameters like the population size, and the probability of mutation and crossover. However further investigation is needed to analyze the sensitivity of the engine resulting solutions due to variations in RGA mutation and crossover control parameters in addition to the probability of mutation and crossover. On the other hand, the engine reacts slower for optimizing best configurations for low data rate services compared to high data rate services. This is reflected in terms of higher mean Engine Dynamic Response 
Time compared to high data rate service deployment scenarios.

The use of the Power Limiting Algorithm (PLA) while produces correct solutions (with variable reliability) with minimum greatest allowable transmitted EIRP levels, it does however have its negative impact on the mean Engine Dynamic Response Time which varies according to the Test Case applied.

More future work is still needed to assess the performance of the engine in terms of convergence speeds and dynamic response time compared to conventional GA and other metaheuristic techniques. Moreover to alleviate the problem of the increased mean Engine Dynamic Response Time associated with the Power Limiting Algorithm (PLA); a Learning module as part of the CRE is necessary to educate the $\mathrm{AE}$ as to which maximum allowable transmitted EIRP levels are adequate for a certain scenario based on previous history. Another possibility is to change the utility function to include multiobjective targets such as minimizing BER and minimizing maximum allowable transmitted EIRP levels. A comparison of these approached with our current implementation will help us in the future of choosing the best technique that achieves the best configuration to minimize the link BER with minimum possible transmitted EIRP levels; in the shortest time possible.

\section{REFERENCES}

[1] J. Mitola, III, "Cognitive Radio for Flexible Multimedia Communications", Mobile Multimedia Communications, 1999. (MoMuC '99) 1999 IEEE International Workshop on, pp. $3-10,1999$.

[2] Federal Communications Commission, "Spectrum Policy Task Force," Rep. ET Docket no. 02-135, Nov. 2002.

[3] T. W. Rondeau, Application of Artificial Intelligence to Wireless Communications. PhD thesis, Virginia Tech, 2007.

[4] T. R. Newman, B. A. Barker, A. M. Wyglinski, A. Agah, J. B. Evans, and G. J. Minden, " Cognitive Engine Implementation for Wireless Multicarrier Transceivers," Wiley Journal on Wireless Communications and Mobile Computing, vol. 7, no. 9, pp. 1129-1142, 2007.

[5] Z. Zhao, S. Xu, S. Zheng, and J. Shang, "Cognitive Radio Adaptation using Particle Swarm Optimization," Wiley Journal on Wireless Communications and Mobile Computing, vol. 9, no. 7, pp. 875-881, 2009.

[6] S. Haykin, "Cognitive Radio: Brain-Empowered Wireless Communications," IEEE Journal on Selected Areas in Communications, vol. 23, no. 2, Feb. 2005.

[7] L. D. Davis and M. Mitchell. Handbook of Genetic Algorithms. Van Nostrand Reinhold, New York, 1991.

[8] X. Yang. Engineering Optimization: An Introduction with Metaheuristic Applications. John Wiley \& Sons, Inc. 2010.
[9] Z. Michalewicz, "Genetic Algorithms, Numerical Optimization and Constraints," Proceedings of the Sixth International Conference on Genetic Algorithms, pp. 151-158, 1995.

[10] P. Kaelo and M. M. Ali, "Integrated Crossover Rules in Real Coded Genetic Algorithms," European Journal of Operational Research, vol. 176, no. 1, pp. 60-76, Jan. 2007.

[11] R.A.E. Makinen, J. Periaux and J. Toivanen, "Multidisciplinary Shape Optimization in Aerodynamics and Electromagnetic using Genetic Algorithm," International Journal for Numerical Methods in Fluids, vol. 30, no. 2, pp. 149-159, 1999.

\section{AUTHOR'S PROFILE}

Ramy A. Fathy; received the M.Sc. Degree in Electrical Engineering from Ain Shams University, Cairo, Egypt in 2007. Currently he is with the Egyptian National Telecommunication Regulatory Authority (NTRA) responsible resposible of setting the digital services policies in the Egyptian market. Eng. Fathy is a candidate for the Ph.D. degree in Electrical Engineering, Electronics and Communications Dept., Ain Shams University, Cairo, Egypt. His technical experience covers signal processing, video compression, and DSP related applications specifically those of real time systems, and cryptography. He published several papers in specialized conferences and periodicals in addition to one book in Electronics. His current research interests include cognitive radio, AI, and meta-heuristic techniques.

Ahmed Ali AbdelHafez; received the B.S. and M.Sc. in Electrical Engineering from Military Technical College (MTC) in 1990, 1997 respectively, and his Ph.D from School of Information Technology and Engineering (SITE), University of Ottawa, Ottawa, Canada in 2003. Dr. AbdelHafez is the head of the Cryptography Research Center (CRC), Egypt where he is leading many applied researches in communication security field. He is a visiting lecturer in Communication Dept. MTC, and other universities in Egypt. His research interests include wireless networks and data security, mathematical cryptography and provable security.

Abdelhalim Zekry; graduated from Cairo University in Egypt in 1969. He was offered the M.Sc. degree in 1973 from the same university. He worked as a scientific coworker at TU Berlin, where he got his $\mathrm{PhD}$ at 1981 . He worked as an assistant prof. at Ain-Shams University (ASU), Egypt in 1982. From 1998 till 2004, he moved to King Soud University where he became a professor of Electronics. Now, he is a professor of Electronics at the faculty of Engineering, ASU, Egypt. Prof. Zekry published 133 papers in specialized conferences and periodicals in addition to three books in Electronics. Now, he is driving research on electronics for communication especially the implementation of advanced communication standards using DSP platforms. 


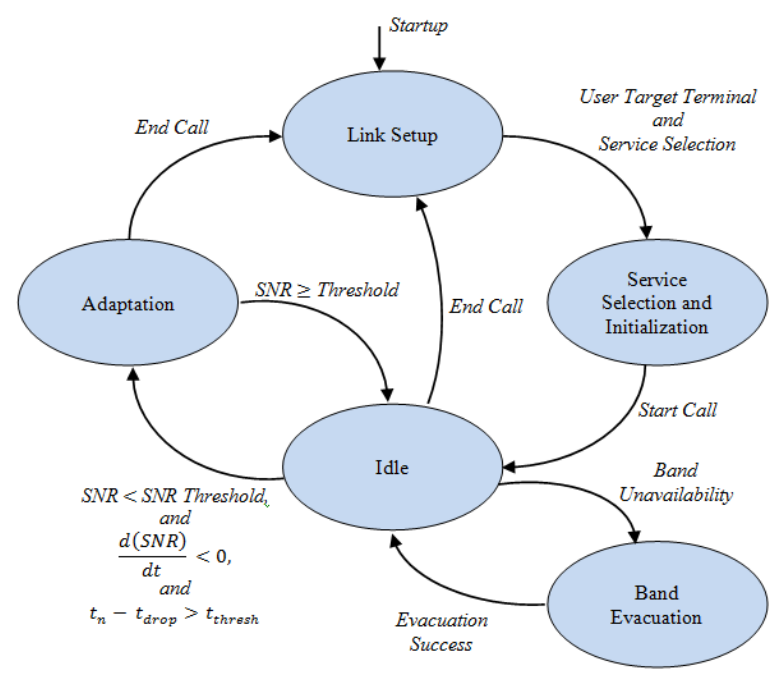

Fig. 1. Cognitive Radio Adaptation Cycle

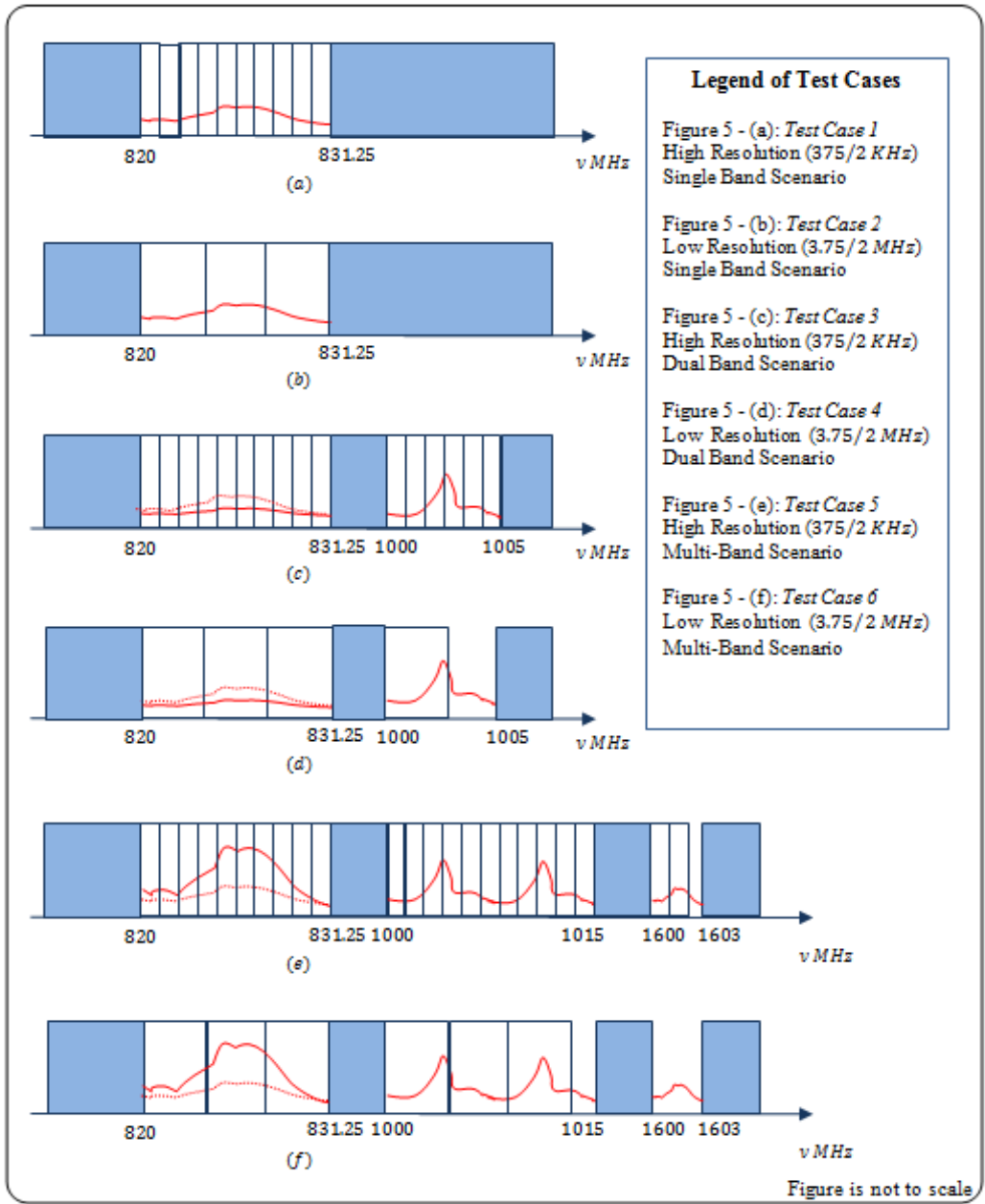

Fig. 3. Test Cases Schematic Diagram for the AE Accuracy Validating

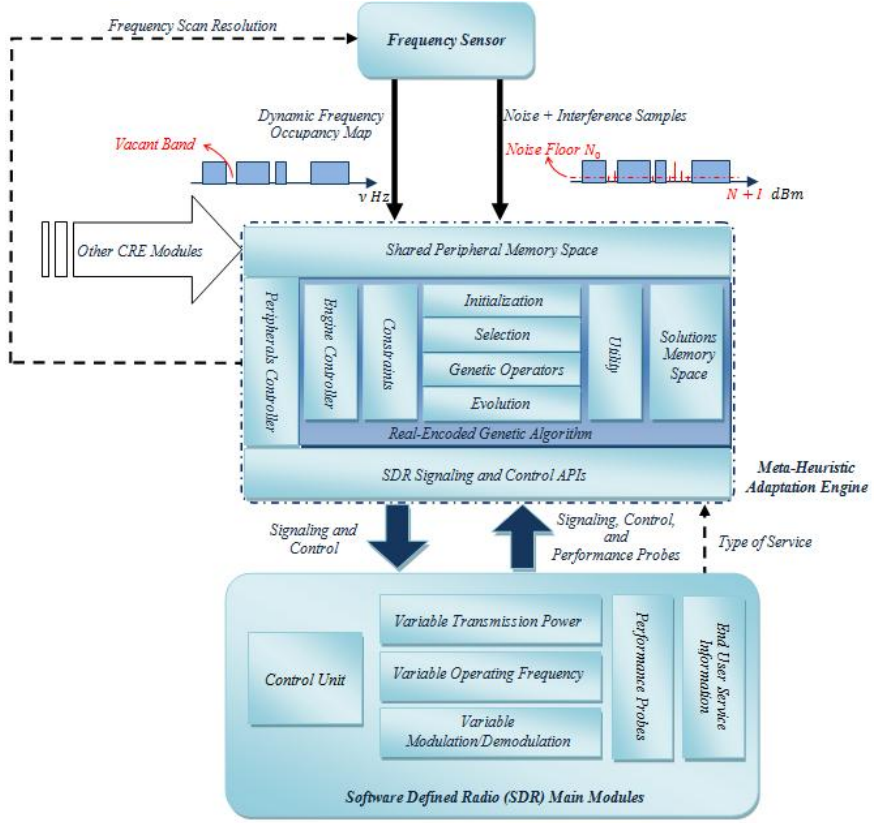

Fig. 2. Proposed AE Architecture
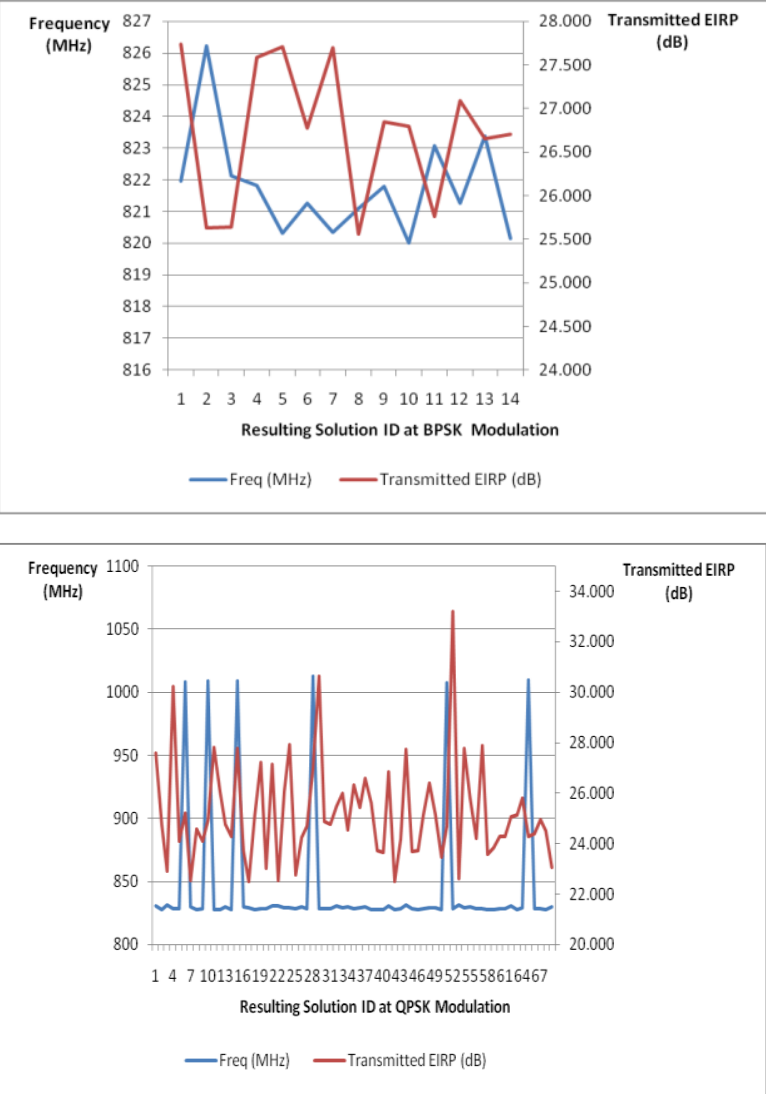

Fig. 4. Sample of the Engine Solutions for Test Case 6 